\title{
Survey of Quadruped Robots Coping Strategies in Complex Situations
}

\author{
JingYe He, JunPeng Shao *, GuiTao Sun and Xuan Shao
}

Key Laboratory of Advanced Manufacturing and Intelligent Technology, Ministry of Education, Harbin University of Science and Technology, Harbin 150080, China; HeJingYescience@163.com (J.H.); sunguitao86@163.com (G.S.); xuanshao84@163.com (X.S.)

* Correspondence: sjp566@hrbust.edu.cn

Received: 6 November 2019; Accepted: 25 November 2019; Published: 27 November 2019

\begin{abstract}
As an important branch of mobile robots, quadruped robots have high flexibility, high adaptability, and high dynamics, which provide excellent maneuverability and environmental adaptability. In the past ten years, researchers have done a lot of research on the ability of the quadruped robot to cope with the complex environment and published many results in order to make the working environment of the quadruped robot closer to reality. This paper collected these research results and divided these literatures into three categories according to different situations: crossing challenging terrain, walking on slope, and coping with interference, respectively, introducing representative methods. The purpose of this review is to summarize and analyze the previous research results and provide guidance for future research on quadruped robots in complex situations.
\end{abstract}

Keywords: quadruped robots; challenging terrain; slope walking; interference; survey

\section{Introduction}

The legged robot is an important branch of the mobile robot field. When compared with the tracked or wheeled robot, the foot robot has better flexibility and terrain adaptability. The advantage of the quadruped robot is that it is simpler than the hexapod robot and has better stability and load capacity than the biped robot. It can be applied to geological exploration, Post-disaster reconstruction, disaster relief, and other complex environments due to its excellent dynamic stability and adaptability in complex environments.

Since the 1980s, the Raibert team at the MIT-Leg lab has conducted extensive research on quadruped robots and it has presented a number of classical theories that influence subsequent research. For example, in 1984 [1], the control of the two-dimensional single-legged jumping robot was decomposed into three parts: vertical jump control, horizontal speed control, and body attitude control. In 1986 [2], the control of dynamic legs was simplified by symmetrizing the variables during contact between the foot and the ground. In the same year, the proposed virtual legs theory [3], which made extending the single-foot control algorithm to multi-foot gait control, such as trot, pace, and bound possible. Through the above theoretical basis, the quadruped robot has rapidly developed in these decades. At present, the representative Quadruped robots are: BigDog [4], LittleDog [5], and Spotting [6] from Boston Dynamics; ANYmal [7] from Swiss Federal Institute of Technology Zurich; and, HyQ [8] from the Italian institute of technology.

Gait planning that is based on the kinematics model is a traditional method of gait planning for quadruped robot. Sakakibara [9] proposed a periodic foot trajectory, reducing the initial impact force between the foot and the ground, and experimental subject walked on a flat terrain at a speed of $2.5 \mathrm{~km} / \mathrm{h}$, based on the wave motion of a cam. On this basis, LiPeng Wang [10] proposed an optimization algorithm to solve the contact impact during walking and planned the impact-free foot trajectory. 
Guoteng Zhang [11] controlled the joint output moment to achieve compliance leg control and planning trot gait. YiBin Li [12] carried out the trot gait planning through the forward kinematics and inverse kinematics, and calculated the torque output of the joint through the inverse dynamics, so that the experimental subject successfully walked on the flat terrain. Jian Meng [13] modified the classic SLIP model, while using a vertical virtual spring-damper model in the contact buffer phase, using the virtual model to adjust the hip joint position of the virtual leg in the thrust phase and using the Bezier curve to plan the foot trajectory to complete the planning of bound gait.

Mstsuoka [14] proposed the central pattern generator (CPG) controlled method to simulate the biological spontaneous rhythmic movement. The CPG control was mainly based on the simplified single-layer CPG model with some feedback to realize gait control. It cannot only control the gait of quadruped robot, but also control bipedal [15], hexapod [16], and snake-like robot [17]. Huashi Li [18] has constructed two kinds of CPG control networks that can simultaneously the hip joint and knee joint of quadruped robot based on the Hopf oscillator. The designed control network integrates the control of the knee joint and it can output many classic gait control signals. AA Saputra [19] combines the CPG model with the trajectory-based model to separate the motion pattern generation from the swing trajectory generation and generate an effective motion pattern at a certain speed by using a multi-objective evolutionary algorithm (MOEA). Boxing Wang [20] constructed a multi-module controller that was based on CPG. Different parallel modules can superimpose motion. Complete specific control motion through basic motion and superposition motion.

Based on the model control method and CPG controlled method, the quadruped robot can achieve static walking and dynamic walking in flat terrain. Through sorting out the data, it is found that the research in this aspect has been relatively mature, and some people have made a detailed summary and induction of these methods [21]. However, most of these studies are conducted in experimental environments where the work environment is relatively simple. In the real environment, the terrain includes obstacles, gaps, slopes, and other factors. The working environment might also interfere with the robot. Through the sorting and analysis of the literature, it is found that the quadruped robot cannot reach a satisfactory working state in a complex environment. Therefore, it is very important to improve the ability of the quadruped robot to cope with complex situations. This paper summarizes the successful methods of quadruped robots that travel through challenging terrain, slope walking, and anti-interference in recent years, providing ideas for future research work. The following contents of the article are as follows: Section 2 will introduce the method of traveling through challenging terrain; Section 3 introduces the method of walking on the slope; Section 4 is the method of coping with the self-disturbance and external impact during the walking process; and, Section 5 will draw the conclusion and put forward some prospects and suggestions for the robot to cope with the complex situation.

\section{Traveling Through Challenging Terrain}

In recent years researchers have been engaged in the research of the quadruped robot traveling the terrain that includes gravel, steps, gaps, and other obstacles in order to make the quadruped robot complete the tasks in the real world. Xuedong Chen [22,23] designs a fuzzy controller that is based on adaptive resonance theory, which can realize real-time positioning in walking environment by dead-reckoning for the quadruped robot. TITAN can walk in a complex environment by looking for the turning point in the walking environment and the continuous omni-directional gait. But the complexity of the environment is relatively simple. Quadruped robots have the advantages of choosing safe footholds, while using leg joints to move around obstacles, making the posture of the body adapt to the terrain, maintaining the balance of the body, thus traveling challenging terrain when compared with wheeled robots. The robot first needs to understand the environment to select a safe landing point in order to plan the cross trajectory. Once the motion is planned, it needs to execute stably and compensate the disturbance to prevent the deviation from the planned path. The robot should have the ability to re-plan the trajectory when the pre-planned trajectory cannot be performed. 


\subsection{Free Gait Planning}

In the study, the point that cannot provide support in the terrain is called the forbidden zone. For the challenging terrain with the forbidden zone, if the quadruped robot adopts the periodic static gait, it will often lead to the locked state, which makes the robot unstable and the working space limited, and even unable to continue walking. Instead of using the non-periodic free gait, these situations can be avoided. McGhee and frank [24] analyzed the free gait in detail, and then the researchers used the free gait to undertake considerable research through the challenging terrain.

Figure 1 shows the framework proposed by Alexander W. Winkler [25]. An environment model is built online and on-body, while using an effective occupied grid representation. Use Any-time-Repairing $\mathrm{A}^{*}\left(\mathrm{ARA}^{*}\right)$ to search for possible action trees (using the tree structure to store the state of the robot can greatly reduce the complexity of the space), select the rough body path, modify the search area position, and swing leg sequence according to the corresponding action. The four-legged supporting phase is inserted in the method of not explicitly generating the Zero Moment Point (ZMP) trajectory to find the center of gravity (CoG) trajectory that conforms to the stability constraint [26], so that the robot can start the swing leg sequence in any order, in order to improve the robustness of the system. The n-step forward optimization of the body trajectory generates natural full-body dynamic motion. A combination of floating-base inverse dynamics controller and virtual model control is used to accurately perform the planned motion. The experiments on HyQ have shown that the frame can smoothly pass the stepping-stone terrain and cross the interval of $35 \mathrm{~cm}$.

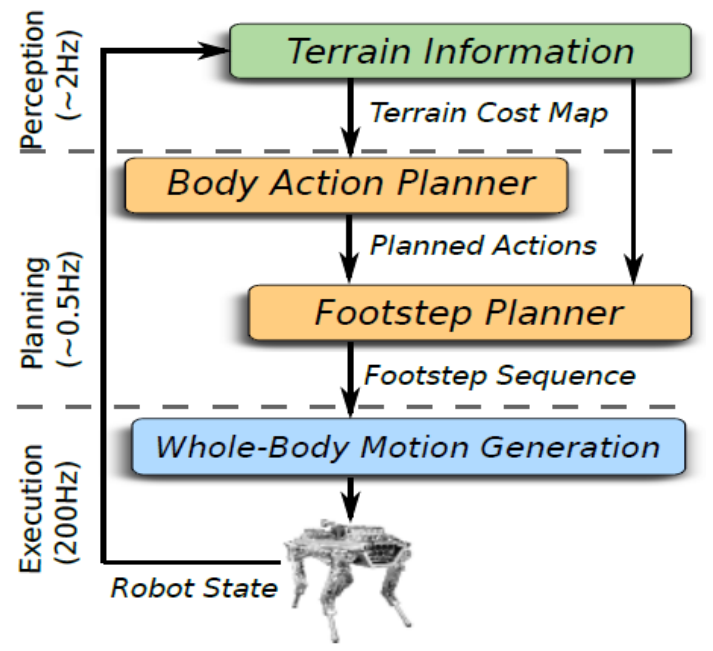

Figure 1. The planning system of sensing terrain information.

Mastalli [27] proposes a motion trajectory optimization framework for rough terrain. While considering the terrain condition and the unfixed phase duration, the center of mass (CoM) motion optimization is combined with the foothold position. Using low-dimensional parametric models to generate a variety of different motion behaviors, combining these models with stochastic-base exploration and receiving the horizon planning, can automatically synthesize the required behavior. The framework can provide robust motion planning without warm start, dealing with local minimum problems that are related to different terrain conditions and walking patterns. First, a series of control parameters (pressure center displacement, phase duration, and foothold position) were optimized according to the terrain cost map. With a series of parameter preview models and terrain elevation maps, CoM trajectory and swing leg trajectory are simultaneously generated. Finally, the body controller and joint space torque controller jointly calculate torque command to realize low-dimensional planning. In addition, the estimated support plane and the maximum allowable angular acceleration of the body are used to adjust the posture and height of the body to adapt to the change of terrain elevation. HyQ tested this method through various challenging terrains. The experimental success rate of the 
stepping-stone was $90 \%$, which proves that the method can improve the operability of the system in practical environment applications.

Zhang $[28,29]$ proposed a continuous free gait for crossing the challenging terrain, which included the forbidden zone. Each gait cycle is divided into three phases: motion planning phase (MPP), body sway phase (BSP), and foot swing phase (FSP). The foot with the smallest motion margin is selected as the following swing foot in order to ensure that the robot can move forward continuously and reduce the lateral motion of the robot in the process of walking to improve the stability. CoG trajectory is generated by static stability criterion to simplify the complexity of gait planning method. When the four feet are on the ground at the same time, the body swing movement (BSM) is used to guide the COG to the centroid of the supporting triangle to ensure a sufficient stability margin during the movement. The forbidden zone and candidate foothold position of the terrain are obtained by processing the point cloud data of challenging terrain. The optimal foothold position is both satisfying the longest step length and make after following swing foot generate the maximum supporting triangle. The simulation results show that the robot automatically advances at a constant speed along the expected direction on the challenge terrain.

\subsection{Hierarchical Control Planning}

Free gait planning is mostly based on the search of foothold position. The disadvantage is that, when the planning path is long or there are many planning parameters, the search space becomes larger, which leads to the increase of planning time and control system tasks. The researchers adopt hierarchical control structure to simplify the control framework in order to solve the complexity and difficulty of the control framework. Different levels of planners and controllers are independent of each other. Finally, they cooperate with each other to form a complete control system to control robot motion.

Kolter [30] proposed hierarchical control architecture on Little Dog, dividing the control system into high-level planner, low-level planner, and low-level controller, as shown in Figure 2. The high-level planner is used to determine a set of footsteps across the terrain, which is robust to slight slippage and deviation. The low-level planner plans the desired trajectory for the moving foot and the robot's CoG to achieve the upcoming footsteps while maintaining static stability. The function of the low-level controller is to detect stability and recovery, body stability, and closed-loop foot placement. The planned trajectory can be executed with slight disturbance and slip. The experiments show that this method can make LittleDog walk stably on the challenging terrain, where the height of obstacles is the same as the leg length of the LittleDog.

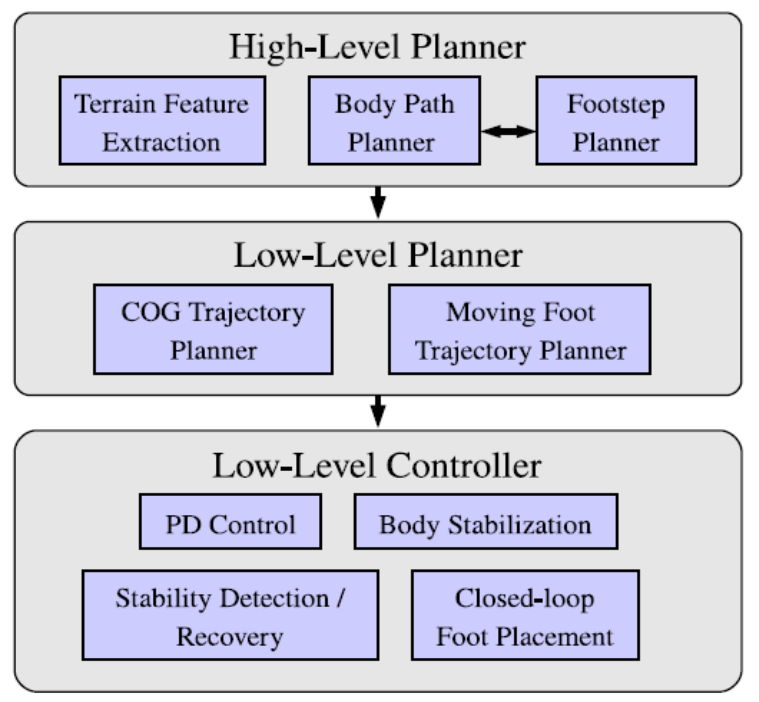

Figure 2. Hierarchical control architecture on Little Dog. 
Guanchu Liu [31] decomposes the problem of planning free gait crossing obstacles into high-level free gait sequence planning that is based on a * search algorithm and low-level obstacle crossing footstep planning based on step time and joint energy consumption comprehensive optimization. Firstly, the off-line expert teaching algorithm was used to learn the experimental terrain to obtain the foothold evaluation. Subsequently, based on this, plan the moving path through the challenging terrain. In the process of moving, online search the foothold position of the next few steps based on the minimum foothold cost. In the simulation, the body posture of the quadruped robot is relatively stable, and there is no big impact when the foot contacts the ground, so it can successfully pass the challenging terrain.

The hierarchical framework that was proposed by Wermelinger [32] establishes traversable maps by calculating the typical topographic features on elevation maps, such as slope, roughness, and step height. The traversable map is used to evaluate the traversability of the robot in the relevant position. Path planning adopts the RRT* algorithm. The hierarchical planning architecture ensures that the paths can be re-planned when new environment data are perceived during path execution. The global path will significantly change when terrain information updated because the robot has no prior knowledge, but the short-range planner ensures the local validity of the path. The framework was integrated on Starlet for experimentation. StarIETH can use static gait to cross challenging terrain, including ramps and large obstacles that must be bypassed, as shown in Figure 3.

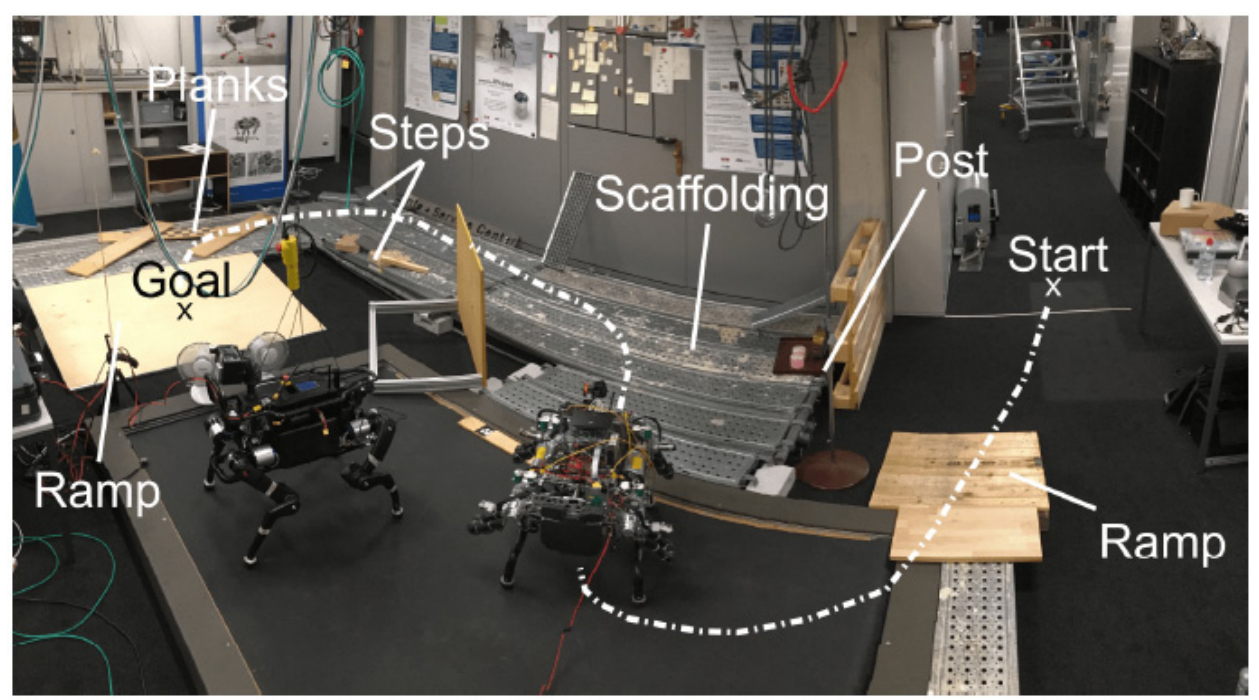

Figure 3. StarIETH use hierarchical framework cross challenging terrain.

Fankhauser [33] proposed a motion planning method for quadruped robot to perceive rough terrain. The elements of terrain rendering, state estimation, control, and planning are combined into an integrated system that can be performed in real time on the robot. The method consists of several modules that are interconnected, but run in parallel at different update speeds, as shown in Figure 4. First, create a series of footsteps that are based on target posture and current leg standing position. Secondly, the next feasible foothold is found by the binary foothold scores map (in the elevation map, the safe foothold point is 1 , the instability point is 0 ) and the attitude optimizer. Finally, once a suitable foothold is found, a collision-free trajectory that connects the current foot position and the selected foothold can be designed based on the Signed Distance Field (SDF) of the terrain. As the map is constantly updated, performing the pre-planned step and the next step planning simultaneously. The motion planner is executed at each step in order to adapt to terrain changes and disturbances. The experimental results show that ANYmal can successfully climb steps and obstacles (the height of obstacles is $38 \%$ of the length of legs). 


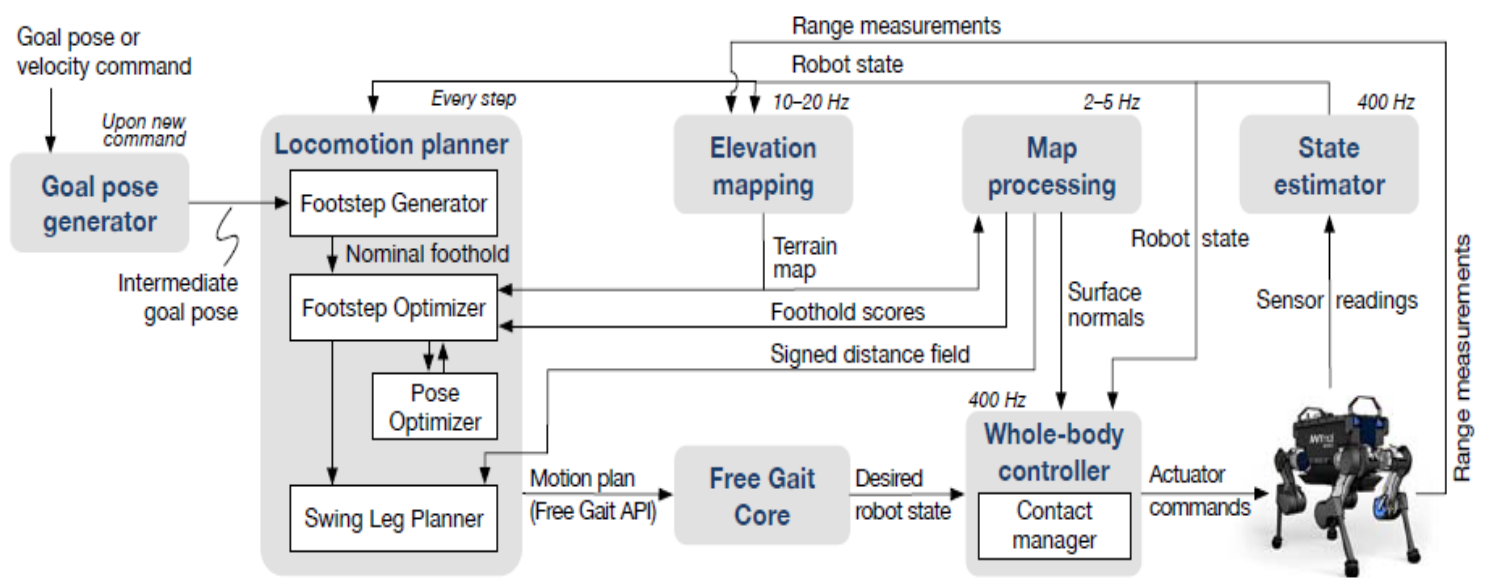

Figure 4. Hierarchical control system on ANYmal.

Li [34] designed a hierarchical control system for the quadruped robot. Firstly, a cost map is constructed by calculating the topographic features below the robot body and the cost of choosing the default foothold location. The heuristic $A *$ algorithm is used to search the trajectory of the robot center in two-dimensional space. Subsequently, the state of the robot is defined from two aspects: the foothold and the attitude of the robot. Along the robot center trajectory, the state sequence is recursively searched to generate the static gait. These states are stored in a tree structure in order to reduce the space complexity. A typical quadruped robot is designed to verify the effectiveness of the algorithm. The reliability of the method is proven in the simulation environment.

In addition to the above methods, many researchers use the CPG control method to cross complex terrain $[35,36]$. First of all, the CPG model itself is very complicated. Secondly, the motion mode and motion performance of the robot are determined by the control parameters of the CPG model. The adjustment of the control parameters is also a complicated process. Therefore, there is no detailed CPG control method in this paper. Some researchers also try to use dynamic gait to cross challenging terrain. Shkolnik [37] has improved the Rapidly Exploring Random Trees (RRTs) [38] planning framework, LittleDog uses the improved framework to perform bounding gait on challenging terrain, but the complexity of the terrain is lower than other studies. To sum up, the focus of current research is the use of hierarchical framework to plan free gait. The focus of future research should be to use dynamic gait to cross the challenging terrain, so it is necessary to solve the dynamic balance of the body and improve the efficiency of the control framework and the ability to re-plan.

\section{Walking on Slope}

In the real environment, slope topography is a common terrain, so slope walking in recent years is also the focus of research. Quadrupeds are easy to walk on slopes, because they can adjust their posture. However, for quadruped robots, adjusting the body posture according to terrain is a complex problem. Different from traversing complex terrain, the research focus of the slope walking is no longer to choose safe foothold, but to output joint torque, adjust and stabilize body posture, etc. According to the different slope, the walking on slope can be divided into low slope walking and high slope walking.

\subsection{Walking Control Method on Low Slope}

For the common low slope, researchers focus on the stability of the body and whether the foot slips when walking on the slope. This kind of research is mostly limited by the platform hardware and it generally solves these two problems in the model and control mothed.

Shulong Zhang [39] uses the joint sensor and inertial measurement unit (IMU) to estimate the slope angle and the uphill angle. The use of the slope angle and the uphill angle to calculate the pitch and roll angles required keeping the body parallel to the ground. The motion of quadruped robot is 
divided into two parts in order to simplify the model: plane seven-link closed the chain motion in the direction of support line and linear inverted pendulum in the vertical direction of the support line. The force dispersion algorithm and the selection of the foothold are used to achieve the stability of the robot. The inner and outer loop layered controller is designed to control the body height and support line direction velocity and three attitude angles. Space forces control is used for the support legs, and space position control is used for the swing legs. Finally, the closed seven-link chains motion and the inverted pendulum motion are combined according to the motion synthesis theorem. The simulation results show that the quadruped robot can use the control strategy to stably walk on the $5^{\circ}$ slope with the trot gait, as shown in Figure 5.

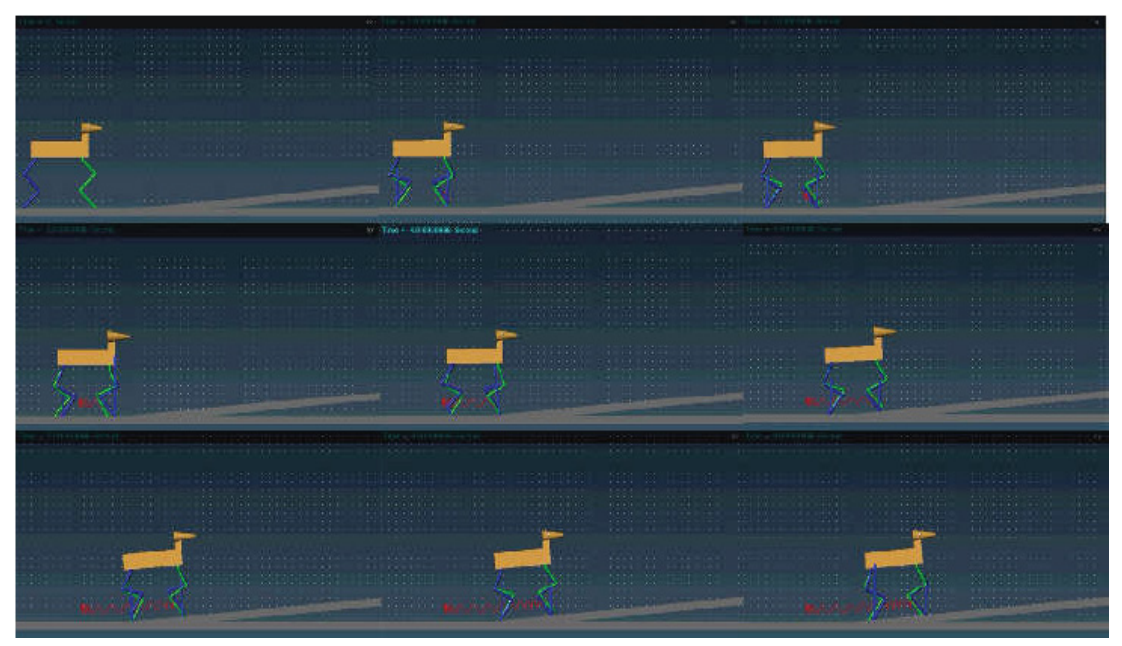

Figure 5. Simulation experiment of quadruped robot uphill movement.

L Wagner [40] proposed a method for avoiding slipping when walking on the slope. Estimating the contact surface normal of each foot only depends on joint torque and measurements from the foot mounted force sensors. The measurement model of the optical force sensor is established. The force sensor on the foot determines the deformation of the foot by optical means, as shown in Figure 6, estimates the contact force of the foot, and combines it with the joint torque measurement in the extended Kalman filter to obtain the minimum tangential force on the contact surface, so as to avoid slipping. Experiments that were conducted at StarIETH show that the method can reliably estimate the contact normal and contact forces of each leg in dynamic motion and achieve stable walking on slopes of $12^{\circ}$ and $17^{\circ}$.

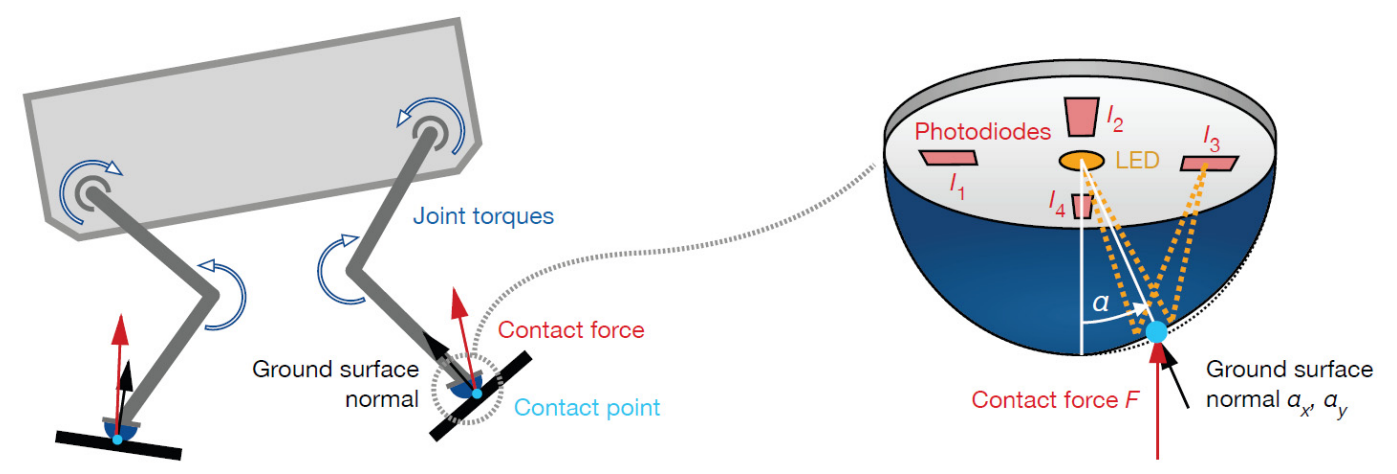

Figure 6. Optical sensor mounted on the foot.

Chengju Liu [41] established a Multi-Layered CPG (ML-CPG) controller based on the semi-center CPG controller in order to solve the shortcomings of the traditional single-layer CPG model in the face of unknown terrain and timely feedback, as shown in Figure 7. ML-CPG is the underlying controller of 
the quadruped robot. By changing the parameters, different motion modes can be generated. A variety of biological reflection models interacting with ML-CPG are established. These models are independent of each other and they correspond to different levels according to functional complexity and real-time performance. In the simulation, the quadruped robot with the ML-CPG controller is adaptable to the unknown slope and it can walk stably on the $12^{\circ}$ slope.

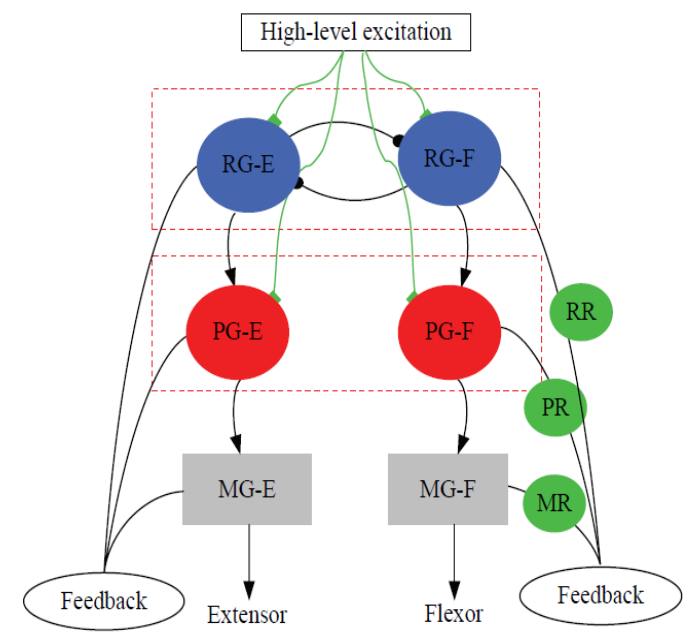

Figure 7. Multi-Layered central pattern generator (ML-CPG) controller.

Chenxiao $\mathrm{Yu}$ [42] proposed a control framework in order to keep the stability of quadruped robot with only IMU sensor when climbing and going downhill, as shown in Figure 8. The control framework consists of a foot trajectory generator and attitude correction controller. The trajectory generator can generate different gait based on the input parameters. The attitude correction controller receives the feedback from the body IMU, and the corresponding adjustment information is obtained through inverse kinematics calculation. The adjustment information is sent to the foot trajectory generator to adjust the joint angle of the leg, so that body posture stability is maintained. The experimental results show that the method can automatically and smoothly adjust the leg joint angle, reduce the body swing, and ensure the stability of the body. The robot can walk stably in a $10^{\circ}$ slope without an obvious fluctuation of the center of mass.

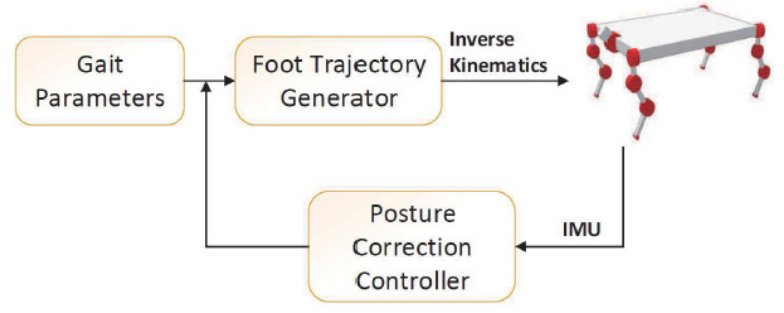

Figure 8. Stable frame with inertial measurement unit (IMU).

\subsection{Walking Mothed on High Slope}

High slope terrain is the difficulty of slope terrain research. Not only has the higher request to the control algorithm, it also has the higher request to the experiment platform hardware. High-precision torque control is the key to solving the problem [43]. Low precision will lead to huge differences between the simulation and experiment. In recent years, advances in theoretical tools $[44,45]$ and the introduction of the quadratic programming $(\mathrm{QP})$ solver has enabled legged robots to handle high slope dynamic tasks.

Righetti $[46,47]$ optimizes the contact force between the robot and the ground in response to unpredictable interference in the environment. By referring to the inverse dynamics that were applied 
to the manipulator [48], an inverse dynamics controller that is suitable for the legged robot is developed. Different from taking contact force control as the main control target, contact force operation in inverse kinematics is completed through torque redundant resolution, so the main goal of this controller is to track the planned trajectory. The controller works in the control stage. The optimal tracking control is created according to the quadratic linear random combination of contact force and command. In addition to optimizing the trajectory during the planning phase, the contact force between the foot and the ground is also optimized. Ensure the minimum contact force when tracking the expected motion path and reduce the foot slip when walking on the challenging terrain. Little Dog successfully walked on a $26^{\circ}$ slope, as shown in Figure 9. It proves that the controller has the advantages of simple structure, high computational efficiency, and good model error robustness.
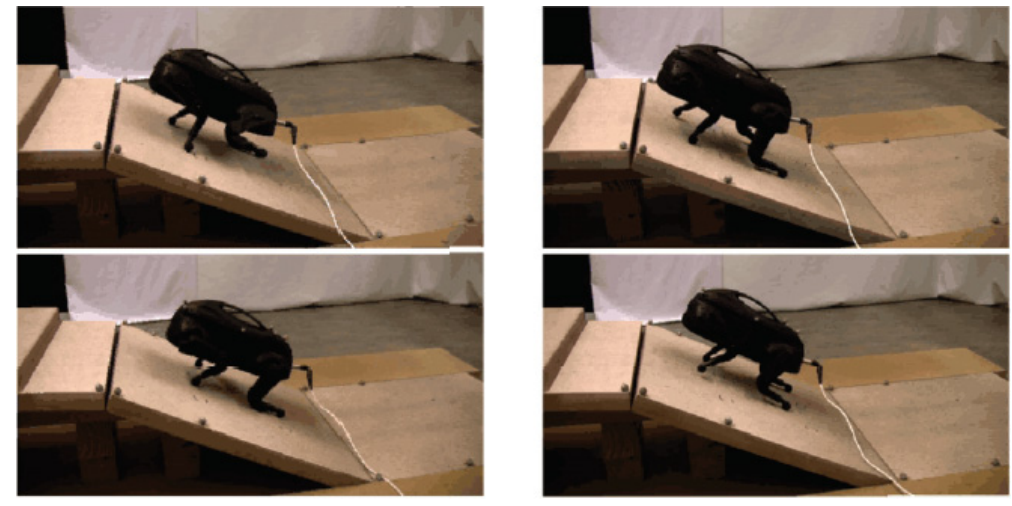

Figure 9. LittleDog walking on $26^{\circ}$ slope.

Marco Hutter [49,50] proposed a hybrid operational space control (OSC) framework that combines leg flexibility control with accurate foothold position control in order to ensure StarIETH smooth walking on high slope terrain. This control method uses the local position control in the low-level controller to perform high-performance tracking task, i.e., swinging leg control, while the rest of the robot interacts with the environment in the inverse kinematics framework to ensure the robustness of the system. Firstly, all of the dynamic tasks are introduced into a priority task space control structure, and the joint position is controlled by inverse kinematics. The frame measures and compensates the influence of position control nodes on torque control components in order to achieve the same task space behavior as pure torque control system. In the experiment, StarIETH used this controller to achieve static stable walking on a $40^{\circ}$ slope, as shown in Figure 10. It proves that the control frame has robustness to irregular terrain and precise foot position control.

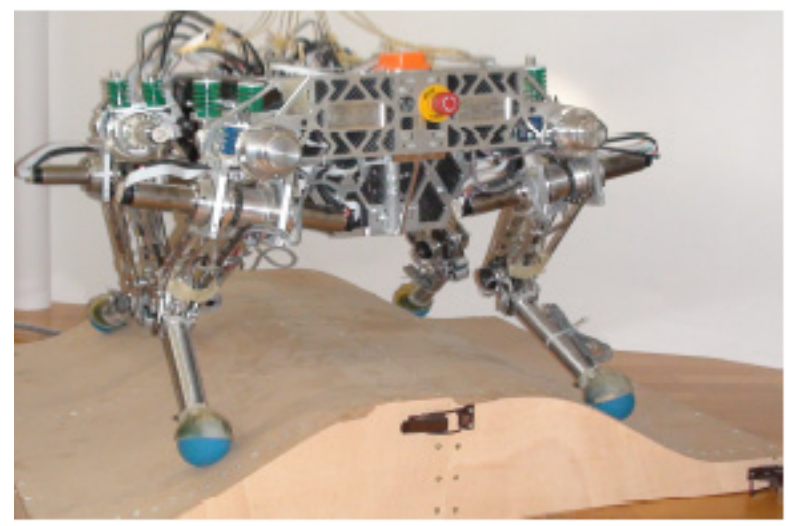

Figure 10. StarIETH walking on $40^{\circ}$ slope.

Focchi [51] proposed a four-legged quasi-static walking framework, including planning and control. The weight of the robot is distributed between the supporting feet to realize the unilateral 
contact force constraint. The QP-base controller is derived from centroid dynamics to avoid the discontinuity of joint torque when the foot establishes or disconnects contact with the ground. The QP cost function is used to reduce joint torque to avoid reaching the joint torque limit. The direct control of the Ground Reaction Force (GRFs) at the support foot gives the desired position of the CoM and the orientation of the robot base. HyQ uses this frame to walk on a $50^{\circ}$ slope, as shown in Figure 11.

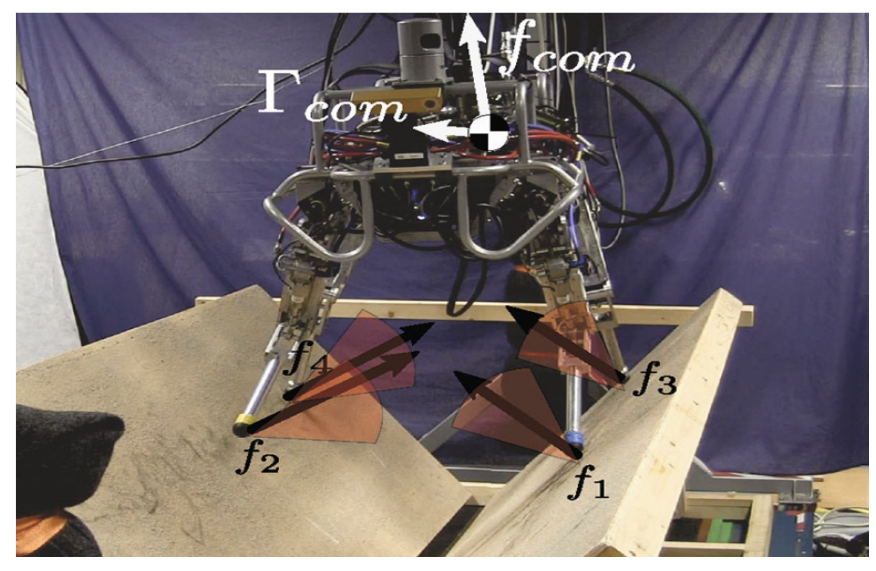

Figure 11. HyQ walking on $50^{\circ}$ slope.

In summary, the contact force control is the basis for ensuring the stability and balance of the robot, whether it is low-slope walking or high-slope walking. The QP solver, combined with modern CPU computing power, can solve small and medium-sized QP problems in the fast control loop, but it is only limited to a few platforms and tasks. The existing experimental slope cannot match the complexity of the real world. Factors, such as low friction, unevenness, and slope change, are the main research directions for slope walking. Pneumatic artificial muscle [52] is a new development of soft brakes. Due to its excellent adjustable stiffness, it can be used in quadruped robots to increase torque control accuracy. Is one of the future development ideas.

\section{Coping With the Self-disturbance and External Impact}

In the process of quadruped robot walking, in addition to considering the problems of foot trajectory planning, foothold position, and contact force between foot and ground, it also has to face self-disturbance and external interference. In the process of moving, the center of mass is always in a floating state, which leads to the disturbance of the body and the impact between the foot and the ground, because the structure of quadruped robots is a strong coupling non-linear system. The external interference includes the change of the robot's load, the impact between the foot, and the ground, the external thrust and the foot slip, etc. Strong self-disturbance will affect the compliance of gait trajectory and excessive external impact will lead to task failure or even hardware damage. Therefore, identifying and coping with interference is essential for quadruped robots to control body stability and attitude recovery. In the current study, researchers will consider the effects of interference in the gait planning method [53-55]. The following will introduce some specific research regarding coping with interference.

The recognition of external disturbances is the basis for quadruped robots to adapt to complex environments. Xinghua Tian [56] proposed an indirect method to identify external interference. While using hydraulic driving force and robot inertia as input, through dynamic model calculation, the external interference is expressed as a 6-DOF force screw (including force and moment) applied to the robot's center of mass. The measured external force can provide data for the following control scheme. Perform the multi-directional pulling experiment on Baby-Elephant. The results show that the external force that is measured by the method is basically the same as the actual external force applied to the body, as shown in Figure 12. 


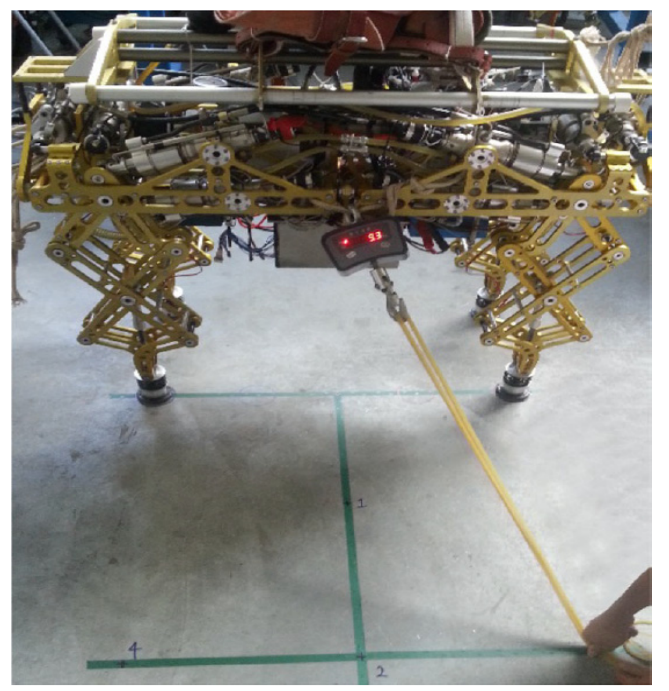

Figure 12. Pulling force applied to the left side of baby-elephant.

The push recovery strategy often simplifies the complex symmetrical movement and structure of the quadruped robot. This simplification inevitably leads to inaccurate calculations. Yang Zhen Chen [57] uses the reinforcement learning (RL) algorithm that can adapt to model errors to solve this problem [58]. Firstly, the simplified quadruped robot model is used to reduce the dimension of action and state space. Subsequently, the prior knowledge based on the simplified model is used to improve the efficiency of the RL algorithm. Through the learning process, the strategy can provide a foot estimation position for the recovery process. In the simulation, this method is compared with the traditional method, and the results show that the quadruped robot has higher recovery efficiency by using the RL algorithm.

Edrisi Farid [59] changed the attitude of the quadruped robot to cope with interference by merging sensor feedback information. This method needs to be implemented with IMU, 3-axis gyroscope, and a 3-axis accelerator. A switching extended Kalman filter (SEKF) is designed to fuse sensory information in order to use the data of these sensors. SEKF can accurately estimate the attitude of the quadruped robot and the gyroscope bias. In this filter, switching rules is used to separate the EKF measurement model of the robot under accelerated or non-accelerated motion. Afterwards, based on the output of observers, support legs are used to compensate for body deviations. In the actual experiment, the robot is placed on the board, which changes the slope with time. The robot can correct the platform interference according to the corrected pitch angle of SEKF output, as shown in Figure 13.
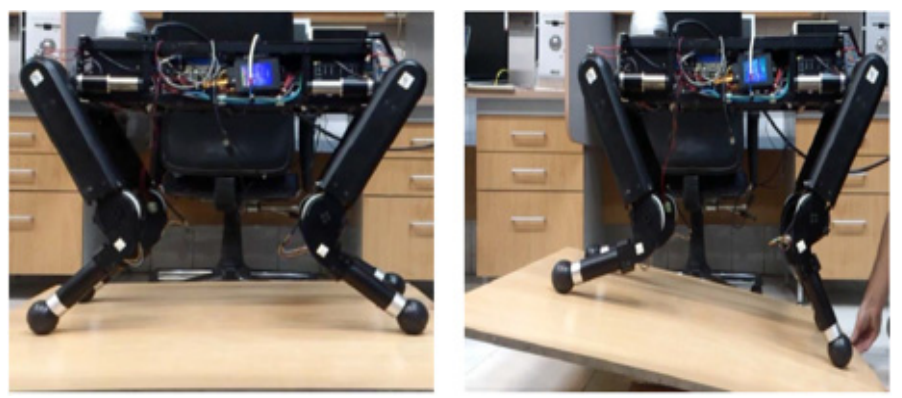

Figure 13. Robot changing attitude to cope with platform interference.

Bruno Floriano uses the multi-objective genetic algorithm to generate gait and optimize the motion parameters of the robot in order to respond to external disturbances [60]. Balance control uses kinematics to correct any interference that might cause instability in the body. Balance control is combined with an empirical method, in which the speed of the joint is proportional to the speed 
of the body and it is measured by an embedded accelerometer. With some specific gains, experience determines the better control of the balance of the robot. Finally, the experiment on the quadruped robot shows that the balance control method that is based on kinematics and empirical methods can correct the interference.

Mahdi Khorram [61,62] proposed a robot balance recovery framework to deal with external unknown interference, as shown in Figure 14. The whole-body dynamics model is used in the framework that enhances the robot's ability to recovery balance with all degrees of freedom. A balance controller is defined based on PD (proportional-derivative) controller, which is used to calculate the acceleration that is needed to restore the robot balance. Stability and slip avoidance conditions are added to the contact force optimization as linear constraints, and the wrenches that are needed to restore stability are calculated. The simulation results show that the framework can restore the balance of the robot under disturbance.

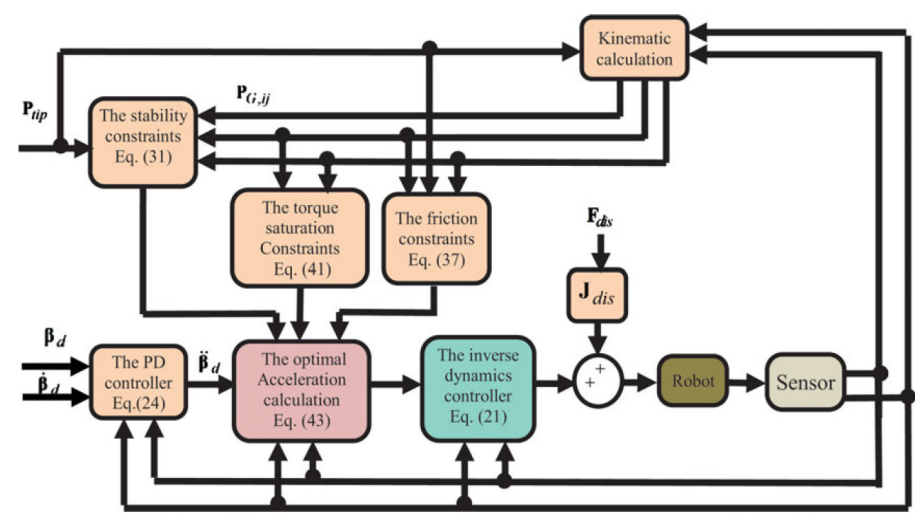

Figure 14. Balance controller defined by PD controller.

Dongyi Ren [63] used the attitude of the body as the feedback to plan the desired foot trajectory in order to suppress the disturbance of the body. Attitude control is based on the D-H (Denavit-Hartenberg) kinematics model to obtain the desired position of the foot. Based on the spring-damping model, the support force of the supporting leg is used as feedback to control the output torque of each joint to restore balance to verify the body disturbance suppression method. Experiments were carried out on a quadruped robot, during which lateral forces were randomly applied to the body. The results show that the pitch angle and roll angle of the robot has little change for the random lateral force applied to the body, and the robot can recover stability within $3 S$.

Takemura [64] has carried out the early work of dealing with the foot slip. Two schemes, long term and short term, are proposed, but they can only be used when the normal of contact surface is correctly estimated or the ground friction is large. Michele Focchi [65] proposed a short-term slip recovery strategy based on the whole-body controller and formulated the controller as a QP problem in order to solve these limitations. Optimize the ground reaction force while implementing the body wrench. When considering that the ground can only exert thrust, a unilateral constraint is introduced to obtain the friction cone limit. By correcting the estimate of the contact surface slope and friction coefficient, the robot is allowed to apply a force that reaches the friction limit and recover from the slip. The experiments have shown that HyQ can perform static walking on highly slippery planes and moderately slippery ramps. This method is only applicable to torque-controlled robots with advanced controllers that can be optimized for ground reaction forces.

The interference recovery that is described above is only applicable when the external impact is not large enough to require a reactive step-by-step strategy. If the external impact is too large, drop control should be adopted [66] to reduce the hardware damage. Whether dealing with self-interference or external disturbances, precise sensors, accurate calculation frames, advanced actuators, and dynamic controls are required. Many platforms cannot achieve the expected experimental results due to the 
limitation of hardware. With the update of hardware, the research on interference response will make great progress.

\section{Conclusions and Prospect}

Some conclusions can be drawn through the above literature summary. The quadruped robot uses the aperiodic free gait to successfully pass the challenge terrain, but at a slower speed. The introduction of the QP solver makes it possible for the quadruped robot to walk on high-slope terrain, but the control algorithm is more demanding. Responding to interference mainly relies on high-precision sensors and advanced actuators, which is a limitation of some existing experimental platforms.

Researchers have conducted extensive research on animal movements in order to enable quadruped robots to cope with complex situations. However, even in biology, the precise factors that determine gait accuracy are inconclusive. There is no uniform standard that affects the gait generation of quadruped robots. So far, most gait generation methods consider energy consumption, mechanical structure, joint torque control, or heuristic structure to optimize gait. These studies provide a large number of useful offline or online tools for the research and development of quadruped robots. In recent years, quadruped robots have greatly improved their adaptability in complex environments due to the update of sensors and algorithms, but they still cannot handle the work in the real world. Improving the speed of crossing challenging terrain, low friction slope walking, and coping with large external forces is the focus of future research. With the high integration of control algorithm, it is also a development direction to integrate various control modes into an online control system. According to different terrains, control modes can be switched, and a reasonable gait can be selected to traverse different terrains, so as to enhance the adaptability of the quadruped robot in the real environment. A new trend of deep neural networks (DNNs) is to provide a surrogate model, semantic segmentation, etc., so that we can attack semantic SLAM field with such robots and solve the multi-task assignment problem of robots. This provides a new research idea for enhancing a quadruped robot to cope with complex situation.

Author Contributions: Investigation, J.H.; resources, J.S. and G.S.; data curation, J.H. and X.S.; supervision, J.S. and G.S.; writing—original draft preparation, J.H.; writing—review and editing, J.H.

Funding: This research received no external funding.

Conflicts of Interest: The authors declare no conflict of interest.

\section{References}

1. Raibert, M.H. Hopping in legged systems-Modeling and simulation for the two-dimensional one-legged case. J. Trans. Syst. Man Cybern. 1984, 14, 451-463. [CrossRef]

2. Raibert, M.H. Running with symmetry. In Autonomous Robot Vehicles; Springer: New York, NY, USA, 1986; pp. 45-61.

3. Raibert, M.H.; Chepponis, M.; Brown, H. Running on four legs as though they were one. J. Robot. Autom. 1986, 2, 70-82. [CrossRef]

4. Raibert, M.; Blankespoor, K.; Nelson, G.; Playter, R. Bigdog, the rough-terrain quadruped robot. IFAC Proc. Vol. 2008, 41, 10822-10825. [CrossRef]

5. Murphy, M.P.; Saunders, A.; Moreira, C.; Raibert, M. The littledog robot. Int. J. Robot. Res. 2011, 30, 145-149. [CrossRef]

6. Niquille, S.C. Regarding the Pain of SpotMini: Or What a Robot's Struggle to Learn Reveals about the Built Environment. J. Archit. Des. 2019, 89, 84-91. [CrossRef]

7. Hutter, M.; Gehring, C.; Jud, D.; Lauber, A.; Bellicoso, C.D.; Tsounis, V.; Hwangbo, J.; Bodie, K.; Fankhauser, P.; Bloesch, M.; et al. Anymal-a highly mobile and dynamic quadrupedal robot. In Proceedings of the International Conference on Intelligent Robots and Systems, Daejeon, Korea, 8-9 October 2016; pp. 38-44.

8. Semini, C.; Tsagarakis, N.G.; Guglielmino, E.; Focchi, M.; Cannella, F.; Caldwell, D.G. Design of HyQ-A hydraulically and electrically actuated quadruped robot. Proc. Inst. Mech. Eng. Part I J. Syst. Control Eng. 2011, 225, 831-849. [CrossRef] 
9. Sakakibara, Y.; Kan, K.; Hosoda, Y.; Hattori, M.; Fujie, M. Foot trajectory for a quadruped walking machine. In Proceedings of the International Workshop on Intelligent Robots and Systems, Towards a New Frontier of Applications, Ibaraki, Japan, 3-6 July 1990; pp. 315-322.

10. Wang, L.; Wang, J.; Wang, S.; He, Y. Strategy of Foot Trajectory Generation for Hydraulic Quadruped Robots Gait Planning. J. Mech. Eng. 2013, 49, 39-44. [CrossRef]

11. Zhang, G. Research on Active Compliance and Trotting Gait Control of a Quadruped Robot. Ph.D. Thesis, Shandong University, Shandong, China, May 2016.

12. Li, Y.; Li, B.; Rong, X.; Meng, J. Mechanical design and gait planning of a hydraulically actuated quadruped bionic robot. J. Shandong Univ. Eng. Sci. 2011, 41, 32-36.

13. Meng, J.; Li, Y.; Li, B. Bound gait controlling method of quadruped robot. J. Shandong Univ. Eng. Sci. 2015, 45, 28-34.

14. Matsuoka, K. Mechanisms of frequency and pattern control in the neural rhythm generators. J. Biol. Cybern. 1987, 56, 345-353. [CrossRef]

15. Liu, C.; Wang, D.; Goodman, E.D.; Chen, Q. Adaptive walking control of biped robots using online trajectory generation method based on neural oscillators. J. Bion. Eng. 2016, 13, 572-584. [CrossRef]

16. Zhong, G.; Chen, L.; Jiao, Z.; Li, J.; Deng, H. Locomotion control and gait planning of a novel hexapod robot using biomimetic neurons. J. Trans. Control Syst. Technol. 2017, 26, 624-636. [CrossRef]

17. Manzoor, S.; Choi, Y. A unified neural oscillator model for various rhythmic locomotions of snake-like robot. J. Neurocomput. 2016, 173, 1112-1123. [CrossRef]

18. Li, H. Biomimetic Locomotion Control Theories and Methods of Quadruped Robot. Ph.D. Thesis, Beijing Institute of Technology, Beijing, China, June 2014.

19. Saputra, A.A.; Kubota, N. Synthesis of Neural Oscillator based Dynamic Rhythmic Generation in Quadruped Robot Locomotion. In Proceedings of the International Electronics Symposium on Knowledge Creation and Intelligent Computing, Nusa Tenggara Barat, Indonesia, 29-30 October 2018; pp. 184-191.

20. Wang, B.; Wan, Z.; Zhou, C.; Wu, J.; Qiu, Y.; Gao, Z. A Multi-module Controller for Walking Quadruped Robots. J. Bionic Eng. 2019, 16, 253-263. [CrossRef]

21. Shao, J.; Ren, D.; Gao, B. Recent advances on gait control strategies for hydraulic quadruped robot. J. Recent Pat. Mech. Eng. 2018, 11, 15-23. [CrossRef]

22. Chen, X.; Watanabe, K.; Kiguchi, K.; Izumi, K. Path tracking based on closed-loop control for a quadruped robot in a cluttered environment. J. Dyn. Syst. Meas. Control 2002, 124, 272-280. [CrossRef]

23. Chen, X.; Watanabe, K.; Kiguchi, K.; Izumi, K. An ART-based fuzzy controller for the adaptive navigation of a quadruped robot. J. Trans. Mechatron. 2002, 7, 318-328. [CrossRef]

24. McGhee, R.B.; Frank, A.A. On the stability properties of quadruped creeping gaits. J. Math. Biosci. 1968, 3, 331-351. [CrossRef]

25. Winkler, A.W.; Mastalli, C.; Havoutis, I.; Focchi, M.; Caldwell, D.G.; Semini, C. Planning and execution of dynamic whole-body locomotion for a hydraulic quadruped on challenging terrain. In Proceedings of the International Conference on Robotics and Automation, Seattle, WA, USA, 26-30 May 2015; pp. 5148-5154.

26. Kalakrishnan, M.; Buchli, J.; Pastor, P.; Schaal, S. Learning, planning, and control for quadruped locomotion over challenging terrain. Int. J. Robot. Res. 2011, 30, 236-258. [CrossRef]

27. Mastalli, C.; Focchi, M.; Havoutis, I.; Radulescu, A.; Calinon, S.; Buchli, J.; Caldwell, D.G.; Semini, C. Trajectory and foothold optimization using low-dimensional models for rough terrain locomotion. In Proceedings of the International Conference on Robotics and Automation, Singapore, 29 May-3 June 2017; pp. 1096-1103.

28. Zhang, S.; Li, Y.B.; Song, R.; Rong, X.; Li, B. A free gait planning method based on the foothold search stratrgy for quadruped robot. In Proceedings of the 18th International Conference on CLAWAR, HangZhou, China, 6-9 September 2015; pp. 461-468.

29. Zhang, S.; Fan, M.; Li, Y.B.; Rong, X.; Liu, M. Generation of a continuous free gait for quadruped robot over rough terrains. Adv. Robot. 2019, 33, 74-89. [CrossRef]

30. Kolter, J.Z.; Rodgers, M.P.; Ng, A.Y. A control architecture for quadruped locomotion over rough terrain. In Proceedings of the International Conference on Robotics and Automation, Pasadena, CA, USA, 19-23 May 2008; pp. 811-818.

31. Liu, G. Research on Free Gait Planning and Control for Quadruped Robot Walking over Terrain that Contains Obstacles. Master's Thesis, University of Electronic Science and Technology, ChengDu, China, May 2015. 
32. Wermelinger, M.; Fankhauser, P.; Diethelm, R.; Krusi, P.; Siegwart, R.; Hutter, M. Navigation planning for legged robots in challenging terrain. In Proceedings of the International Conference on Intelligent Robots and Systems, Daejeon, Korea, 9-14 Octobor 2016; pp. 1184-1189.

33. Fankhauser, P.; Bjelonic, M.; Bellicoso, C.D.; Miki, T.; Hutter, M. Robust rough-terrain locomotion with a quadrupedal robot. In Proceedings of the International Conference on Robotics and Automation, Brisbane, Australia, 21-25 May 2018; pp. 1-8.

34. Li, X.; Gao, H.; Li, J.; Wang, Y.; Guo, Y. Hierarchically Planning Static Gait for Quadruped Robot Walking on Rough Terrain. J. Robot. 2019, 2019, 12. [CrossRef]

35. Barasuol, V.; Buchli, J.; Semini, C.; Frigerio, M.; De Pieri, E.R.; Caldwell, D.G. A reactive controller framework for quadrupedal locomotion on challenging terrain. In Proceedings of the International Conference on Robotics and Automation, Karlsruhe, Germany, 6-10 May 2013; pp. 2554-2561.

36. Ajallooeian, M.; Pouya, S.; Sproewitz, A.; Ijspeert, A.J. Central pattern generators augmented with virtual model control for quadruped rough terrain locomotion. In Proceedings of the International Conference on Robotics and Automation, Karlsruhe, Germany, 6-10 May 2013; pp. 3321-3328.

37. Shkolnik, A.; Levashov, M.; Manchester, I.R. Bounding on rough terrain with the LittleDog robot. Int. J. Robot. Res. 2011, 30, 192-215. [CrossRef]

38. LaValle, S.M.; Kuffner, J.J., Jr. Randomized kinodynamic planning. Int. J. Robot. Res. 2001, 20, 378-400. [CrossRef]

39. Zhang, S.; Ma, H.; Yang, Y.; Wang, J. The quadruped robot adaptive control in trotting gait walking on slopes. In AIP Conference Proceedings; American Institute of Physics: College Park, MD, USA, 2017; p. 020004.

40. Wagner, L.; Fankhauser, P.; Bloesch, M.; Mutter, M. Foot contact estimation for legged robots in rough terrain. In Advances in Cooperative Robotics; WSPC: London, UK, 2017; pp. 395-403.

41. Liu, C.; Xia, L.; Zhang, C.; Chen, Q. Multi-layered CPG for adaptive walking of quadruped robots. J. Bion. Eng. 2018, 15, 341-355. [CrossRef]

42. Yu, C.; Zhou, L.; Qian, H.; Xu, Y. Posture Correction of Quadruped Robot for Adaptive Slope Walking. In Proceedings of the International Conference on Robotics and Biomimetics, Kuala Lumpur, Malaysia, 12-15 December 2018; pp. 1220-1225.

43. Boaventura, T.; Semini, C.; Buchli, J.; Frigerio, M.; Focchi, M.; Caldwell, D.G. Dynamic torque control of a hydraulic quadruped robot. In Proceedings of the international conference on robotics and automation, Saint Paul, MN, USA, 14-18 May 2012; pp. 1889-1894.

44. Macchietto, A.; Zordan, V.; Shelton, C.R. Momentum control for balance. In ACM Transactions on Graphics; ACM: New York, NY, USA, 2009; p. 80.

45. Lee, S.H.; Goswami, A. A momentum-based balance controller for humanoid robots on non-level and non-stationary ground. Auton. Robots 2012, 33, 399-414. [CrossRef]

46. Righetti, L.; Buchli, J.; Mistry, M.; Schaal, S. Control of legged robots with optimal distribution of contact forces. In Proceedings of the International Conference on Humanoid Robots, Bled, Slovenia, 26-28 October 2011; pp. 318-324.

47. Righetti, L.; Buchli, J.; Mistry, M.; Kalakrishnan, M.; Schaal, S. Optimal distribution of contact forces with inverse-dynamics control. Int. J. Robot. Res. 2013, 32, 280-298. [CrossRef]

48. Awan, Z.S.; Ali, K.; Iqbal, J.; Mehmood, A. Adaptive Backstepping Based Sensor and Actuator Fault Tolerant Control of a Manipulator. J. Electr. Eng. Technol. 2019, 14, 2497-2504. [CrossRef]

49. Gehring, C.; Coros, S.; Hutter, M.; Bloesch, M.; Hoepflinger, M.A.; Siegwart, R. Control of dynamic gaits for a quadrupedal robot. In Proceedings of the International Conference on Robotics and Automation, Karlsruhe, Germany, 6-10 May 2013; pp. 3287-3292.

50. Hutter, M.; Hoepflinger, M.A.; Gehring, C.; Bloesch, M. Hybrid operational space control for compliant legged systems. In Proceedings of the Robotics: Science and Systems, Sydney, Australia, 9-13 July 2012.

51. Focchi, M.; Del Prete, A.; Havoutis, I.; Featherstone, R.; Caldwell, D.; Semini, C. High-slope terrain locomotion for torque-controlled quadruped robots. Auton. Robot. 2017, 41, 259-272. [CrossRef]

52. Li, K.; Nuchkrua, T.; Zhao, H.; Yuan, Y.; Boonto, S. Learning-based Adaptive Robust Control of Manipulated Pneumatic Artificial Muscle Driven by H 2-based Metal Hydride. In Proceedings of the 14th IEEE International Conference on Automation Science and Engineering, Munich, Germany, 20-24 August 2018; pp. 1284-1289.

53. Li, J.; Wang, J.; Yang, S.; Zhou, K.; Tang, H. Gait planning and stability control of a quadruped robot. Comput. Intell. Neurosci. 2016, 2016, 13. [CrossRef] 
54. Gonzalez-Luchena, I.; Gonzalez-Rodriguez, A.G.; Gonzalez-Rodriguez, A.; Adame-Sanchez, C.; Castillo-Garcia, F. A new algorithm to maintain lateral stabilization during the running gait of a quadruped robot. Robot. Auton. Syst. 2016, 83, 57-72. [CrossRef]

55. Boussema, C.; Powell, M.J.; Bledt, G.; Ijspeert, A.J.; Wensing, P.M.; Kim, S. Online gait transitions and disturbance recovery for legged robots via the feasible impulse set. J. Robot. Autom. Lett. 2019, 4, 1611-1618. [CrossRef]

56. Tian, X.; Gao, F.; Qi, C.; Chen, X.; Zhang, D. External disturbance identification of a quadruped robot with parallel-serial leg structure. Int. J. Mech. Mater. Des. 2016, 12, 109-120. [CrossRef]

57. Chen, Y.; Hou, W.Q.; Wang, J.; Ma, H. A strategy for push recovery in quadruped Robot based on reinforcement learning. In Proceedings of the 34th Chinese Control Conference, HangZhou, China, 28-30 July 2015; pp. 3145-3151.

58. Fankhauser, P.; Hutter, M.; Gehring, C.; Bloesch, M.; Hoepflinger, M.A.; Siegwart, R.; Hutter, M. Reinforcement learning of single legged locomotion. In Proceedings of the International Conference on Intelligent Robots and Systems, Tokyo, Japan, 3-7 November 2013; pp. 188-193.

59. Edrisi, F.; Majd, V.J.; Attar, M.; Dini, N. Modifying the attitude of quadruped robot body against disturbances via data fusion. In Proceedings of the International Conference on Robotics \& Mechatronics, Tehrān, Iran, 26-28 October 2017; pp. 55-60.

60. De Oliveira Floriano, B.R.; de Freitas Porphirio, C.; Santana, P.H.M.; Borges, G.A.; Romariz, A.R.S. Walking Pattern Design and Balance Control of a Quadruped Platform. In Proceedings of the Workshop on Robotics in Education, Joao Pessoa, Brazil, 6-8 November 2018; pp. 242-247.

61. Khorram, M.; Moosavian, S.A.A. Balance recovery of a quadruped robot. In Proceedings of the International Conference on Robotics \& Mechatronics, Tehrān, Iran, 7-9 October 2015; pp. 259-264.

62. Khorram, M.; Moosavian, S.A.A. Push recovery of a quadruped robot on challenging terrain. Robotica 2017, 35, 1670-1689. [CrossRef]

63. Ren, D.; Shao, J.; Sun, G.; He, J. Torso disturbance inhibition and experiment research of hydraulic quadruped robot. Chin. J. Sci. Instrum. 2019, 40, 163-171.

64. Takemura, H.; Deguchi, M.; Ueda, J.; Matsumoto, Y.; Ogasawara, T. Slip-adaptive walk of quadruped robot. Robot. Auton. Syst. 2005, 53, 124-141. [CrossRef]

65. Focchi, M.; Barasuol, V.; Frigerio, M.; Caldwell, D.; Semini, C. Slip Detection and Recovery for Quadruped Robots. In Robotics Research; Springer: Cham, Switzerland, 2018; pp. 18-199.

66. Yun, S.; Goswami, A. Tripod fall: Concept and experiments of a novel approach to humanoid robot fall damage reduction. In Proceedings of the International Conference on Robotics and Automation, Hong Kong, China, 31 May-5 Jun 2014; pp. 2799-2805. 